\title{
Takotsubo Cardiomyopathy in a Healthcare Worker During the COVID-19 Pandemic: Caused by the Virus or the Demands of the Many Being Placed on the Few?
}

\author{
Mustafa Mohammed ${ }^{1}$, Samer Zakhour ${ }^{1}$, Jasneet Devgun², James Lee², Thomas Keimig ${ }^{3}$, Dee Dee Wang² \\ ${ }^{1}$ Department of Internal Medicine, Henry Ford Hospital, Detroit, MI, USA \\ ${ }^{2}$ Department of Cardiology, Henry Ford Hospital, Detroit, MI, USA \\ ${ }^{3}$ Department of Radiology, Henry Ford Hospital, Detroit, MI, USA
}

Received: 20/10/2020

Accepted: 04/11/2020

Published: $30 / 11 / 2020$ How to cite this article: Mohammed M, Zakhour S, Devgun J, Lee J, Keimig T, Wang DD. Takotsubo cardiomyopathy in a healthcare worker during the
COVID-19 pandemic: caused by the virus or the demands of the many placed on the few? EJCRIM 2020;7: doi:10.12890/2020_002088.

Conflicts of Interests: The Authors declare that there are no competing interests.

Acknowledgements: The authors thank Karla D Passalacqua, PhD, at Henry Ford Hospital for editorial assistance.

This article is licensed under a Commons Attribution Non-Commercial 4.0 License

\section{ABSTRACT}

We present a case of Takotsubo (stress-induced) cardiomyopathy (TCM) in a healthcare worker that occurred during the COVID-19 pandemic. TCM, or broken-heart syndrome, has various presentations. This case sheds light on how stress due to the COVID-19 pandemic may cause cardiac illness in healthcare workers.

\section{LEARNING POINTS}

- The various symptoms indicating Takotsubo cardiomyopathy are characterized and defined.

- Physicians should be aware that stress in healthcare workers may cause heart disease.

\section{KEYWORDS}

Takotsubo, healthcare worker, COVID-19

\section{CASE DESCRIPTION}

A 60-year-old woman presented with chest pressure and dyspnoea on exertion, which had occurred intermittently several days before presentation. She stated that her symptoms had initially begun 1 week before presentation and included dyspnoea on exertion and consistent chest pressure lasting for approximately 15 minutes. The day before presentation, the patient had also experienced diaphoresis and an episode of disorientation.

The patient's medical history included hyperlipidaemia, iron deficiency anaemia, and sickle cell trait. The patient was a healthcare worker who had been working at a senior nursing home living facility for 19 years. Her duties included giving medicines to nursing home residents, checking nursing home residents' vital signs, and assisting nursing home residents with their daily needs. During the COVID-19 pandemic, her place of employment had laid off several workers, resulting in an increase in the patient's job duties. She had been working at least 5 days per week with 8-9 hour shifts and was regularly called on to work extra shifts. At the start of the pandemic, the facility had insufficient personal protective equipment (PPE). Also, many nursing home residents who the patient had known personally for a significant amount of time, had died from COVID-19. Furthermore, many of the patient's co-workers had become infected with SARS-CoV-2 but had continued 
to work because they were not able to take time off for various staffing reasons. The work stressors had changed the patient's personal life during the weeks leading to her illness. She started eating less, sleeping less and not taking good care of her health. She was also becoming more emotional and more easily irritated. Additionally, the patient was stressed by the lack of proper precautions at work and by the lack of knowledge concerning the safe use of PPE.

On admission, the patient's blood pressure was 188/82 $\mathrm{mmHg}$ and her heart rate was 115 beats per minute with a regular rhythm. Laboratory test results indicated brain natriuretic peptide elevation at $318 \mathrm{pg} / \mathrm{ml}$ (reference <50), high-sensitivity troponin I mildly elevated to 24,29 and $27 \mathrm{ng} / \mathrm{l}$ (reference <19), and haemoglobin $11.3 \mathrm{~g} / \mathrm{dl}$ (reference 12-15). A COVID-19 PCR test from a nasal swab was negative, and a chest $x$-ray showed no respiratory disease. An electrocardiogram (ECG) showed sinus tachycardia and left bundle branch block, which had not been observed in a previous ECG performed 9 months prior to presentation (Figs. 1 and 2).

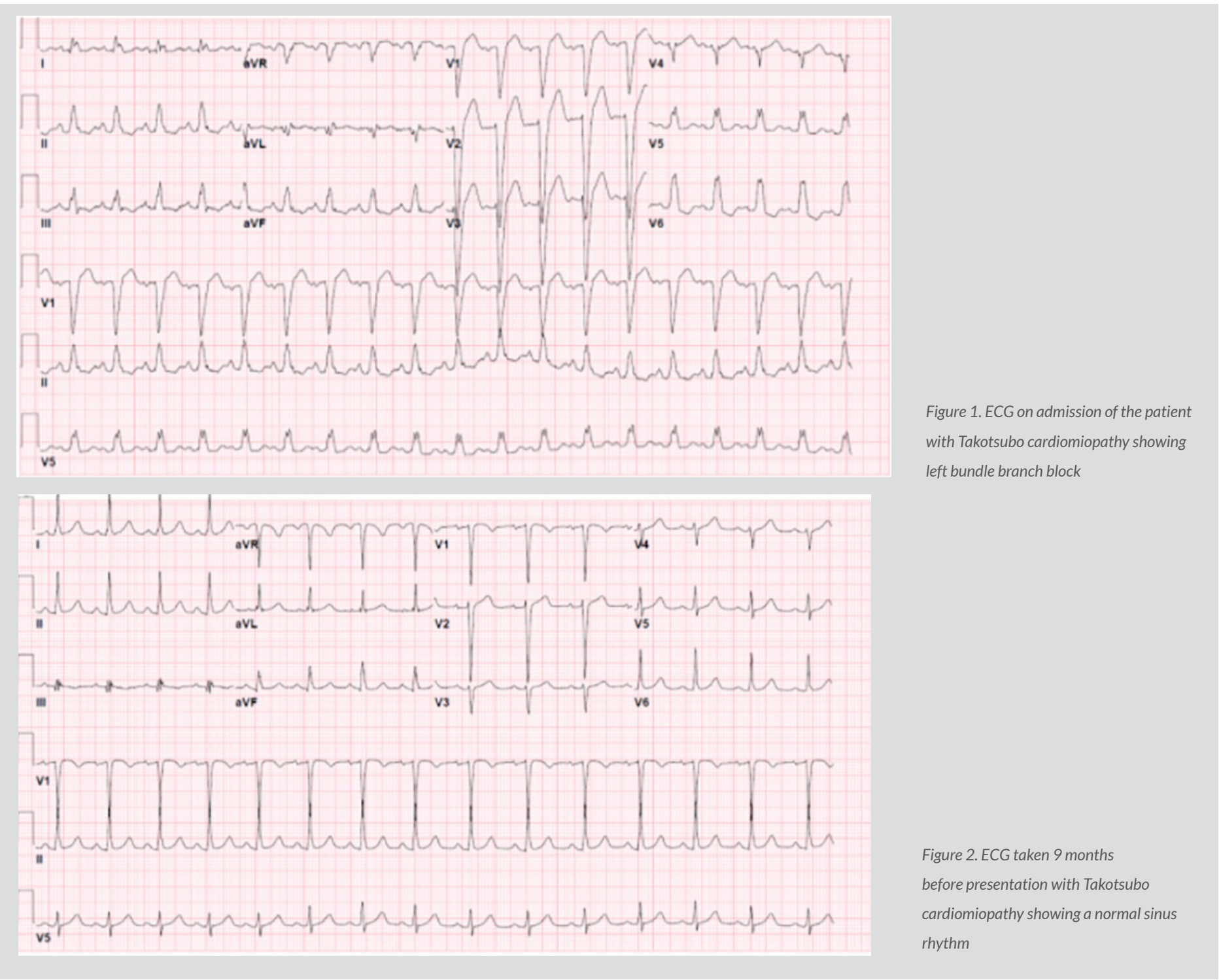

The patient was admitted to the cardiology unit and was treated for non-ST elevation myocardial infarction. A heparin drip was started and the patient was given aspirin and clopidogrel. A transthoracic echocardiogram showed a newly depressed ejection fraction (EF) of $22 \%$, a moderately increased left ventricular (LV) cavity size, and moderate hypokinesis of the mid-distal left ventricular wall with preservation of basal LV contractility (Video 1); all new findings compared to her normal stress echocardiogram one year prior. A coronary angiogram showed normal coronary arteries (Fig. 3A,B). Finally, cardiac magnetic resonance imaging demonstrated global LV hypokinesis with dyssynchronous septal wall motion, an LV EF of 29\%, no abnormal late gadolinium myocardial enhancement, and mitral valve regurgitation (Fig. 3C). 


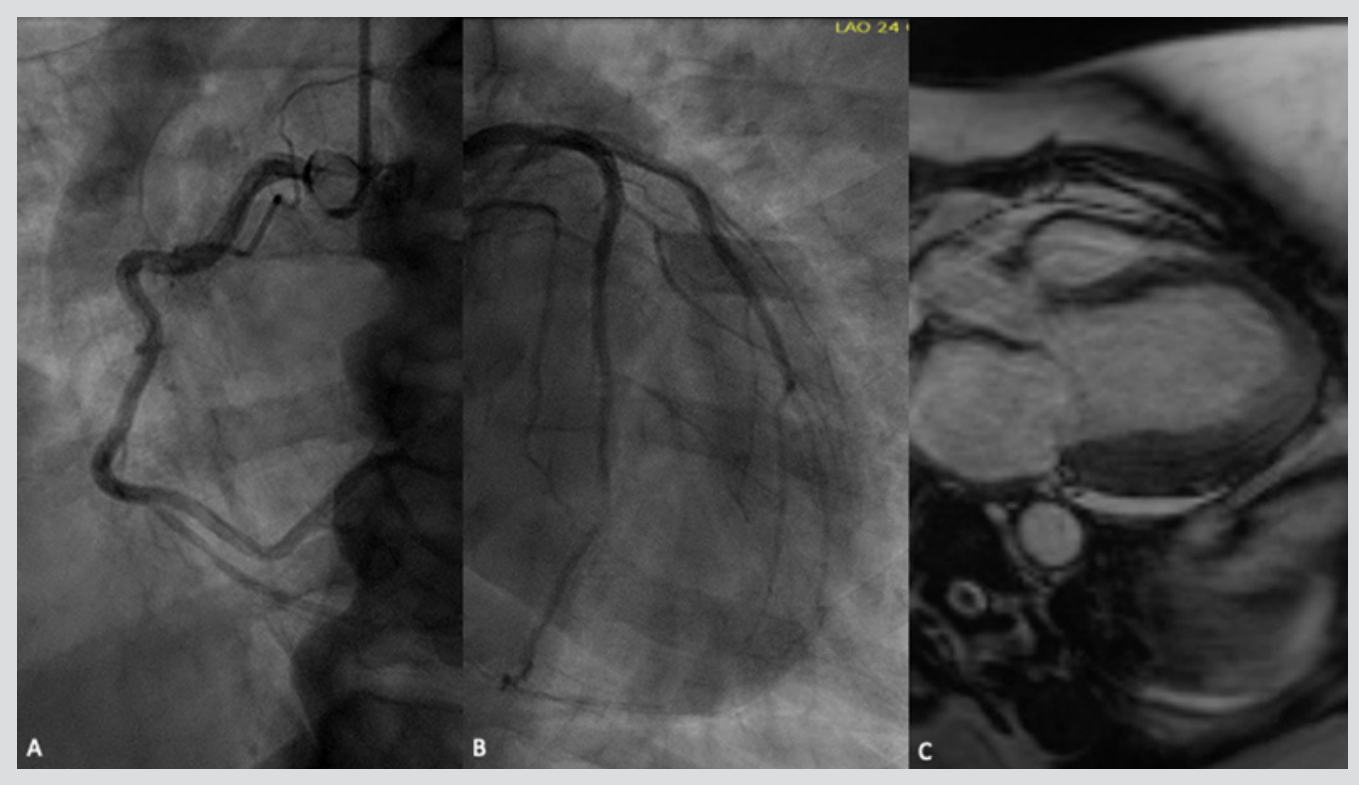

Figure 3. $(A, B)$ Coronary angiogram showing no coronary disease; (C) MRI showing apical ballooning

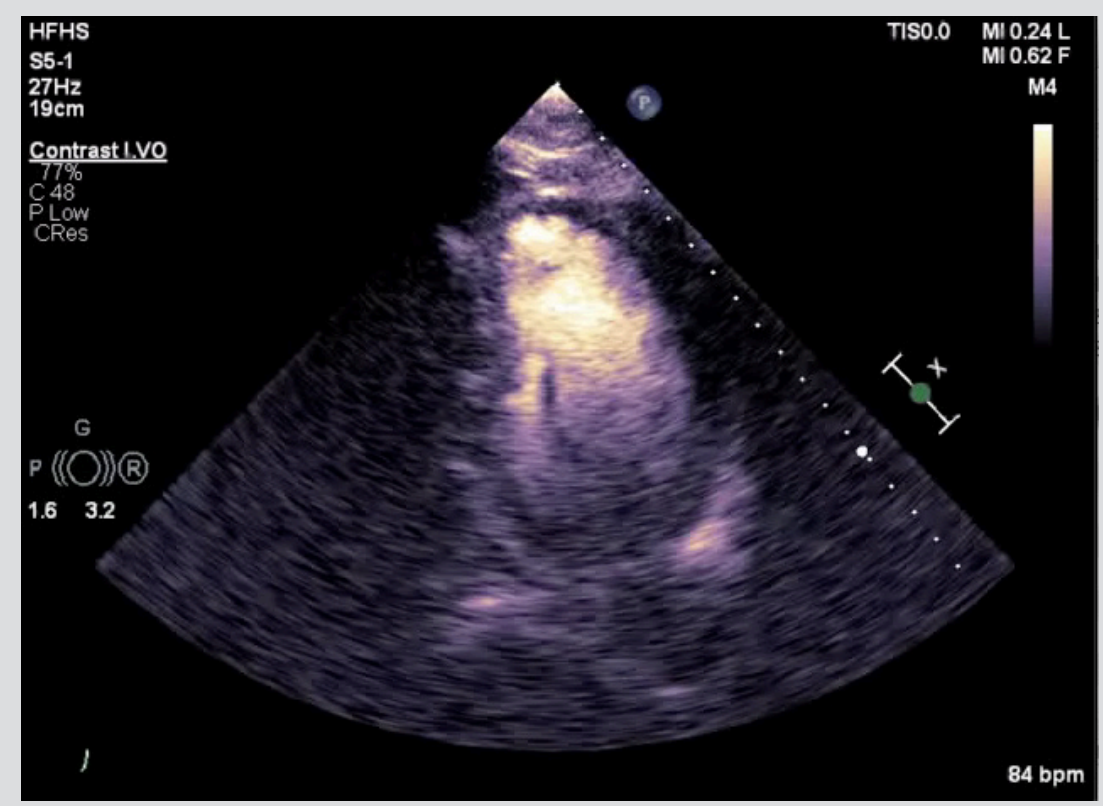

Video 1. Echocardiogram showing reduced ejection fraction with preserved basal left ventricle contractility and mid-distal left ventricle hypokinesis in an apical ballooning pattern Watch here: https://youtu.be/g8HS2OWeXag

The patient was given furosemide, lisinopril, carvedilol and spironolactone, and all were increased as tolerated during her hospital stay. The patient was also given a LifeVest on discharge to wear until her EF is reevaluated on outpatient echocardiogram. Several weeks after admission, the patient's symptoms had resolved, and she continued the same medication regimen.

\section{DISCUSSION}

Although TCM has been described in many studies, the precise cause of this illness remains unknown. Multiple potential causative mechanisms have been described ${ }^{[1]}$, including transient ischaemia from plaque rupture, epicardial spasm, catecholamine-induced microcirculatory dysfunction, and catecholamine toxicity towards cardiomyocytes ${ }^{[1]}$. Researchers have also investigated whether TCM is caused by an inflammatory process ${ }^{[2]}$. However, whether or not TCM is due to inflammation or other processes, it has been firmly established that stress plays a key role in the development of this syndrome.

TCM is most prevalent in women aged 50 years and older ${ }^{[1,3,4]}$. The syndrome presents very similarly to acute coronary syndrome [4,5] and often with elevated cardiac markers or ST-segment elevation ${ }^{[4,5]}$. The reported prevalence of TCM is 5.2 per 100,000 women and 0.6 per 100,000 men in the USA ${ }^{[6]}$. It is important to recognize this syndrome and include it in the differential diagnosis. 
Obtaining a thorough patient history that includes social factors is crucial for early recognition of this condition. The clinical outcome of patients with TCM is generally favourable, with recovery of the LV ejection fraction in most patients ${ }^{[3,4]}$.

Here, we present a case of TCM in a healthcare worker who had been experiencing multiple COVID-19 pandemic-related stresses. This case sheds light upon the stressors that healthcare workers may face during a pandemic. In addition to the physical stress of working long hours, intense emotional stress is also experienced by healthcare workers. These emotional stresses are particularly acute during the COVID-19 pandemic and often include witnessing death on a daily basis. Also, fear of acquiring the disease and fear of potentially infecting colleagues, patients and loved ones, increases anxiety. Recognizing the heavy stress that healthcare workers face and creating stress-reducing programs will be vital for protecting essential caregivers.

\section{CONCLUSION}

It is common in medicine to establish a diagnosis based on medical history and testing alone, without getting to know the patient. This case highlights the importance of learning about a patient's living environment, mental health, and daily stresses. TCM is caused by severe stress, both physical and emotional, and the ability to accurately recognize this pathology relies on the physician's skill in communicating with the patient to uncover the social and emotional factors involved in causing illness.

\section{REFERENCES}

1. Ghadri JR, Wittstein IS, Prasad A, Sharkey S, Dote K, Akashi YJ, et al. Expert consensus on Takotsubo syndrome (Part I): clinical characteristics, diagnostic criteria, and pathophysiology. Eur Heart J 2018;39(22):2032-2046.

2. Wilson HM, Cheyne L, Brown PA, Kerr K, Hannah A, Srinivasan J, et al. Characterization of the myocardial inflammatory response in acute stress-induced (Takotsubo) cardiomyopathy. JACC Basic Transl Sci 2018;3(6):766-778.

3. Templin C, Ghadri JR, Diekmann J, Napp LC, Bataiosu DR, Jaguszewski M, et al. Clinical features and outcomes of takotsubo (stress) cardiomyopathy. N Engl J Med 2015;373(10):929-938.

4. Sharkey SW, Windenburg DC, Lesser JR, Maron MS, Hauser RG, Lesser JN, et al. Natural history and expansive clinical profile of stress (tako-tsubo) cardiomyopathy. Am Coll Cardiol 2010;55(4):333-341.

5. Prasad A, Lerman A, Rihal CS. Apical ballooning syndrome (Tako-Tsubo or stress cardiomyopathy): a mimic of acute myocardial infarction. Am Heart J 2008;155(3):408-417.

6. Deshmukh A, Kumar G, Pant S, Rihal C, Murugiah K, Mehta JL. Prevalence of Takotsubo cardiomyopathy in the United States. Am Heart J 2012;164(1):66-71. 